Received: 15 February 2017

Accepted: 16 May 2017

Published online: 03 July 2017

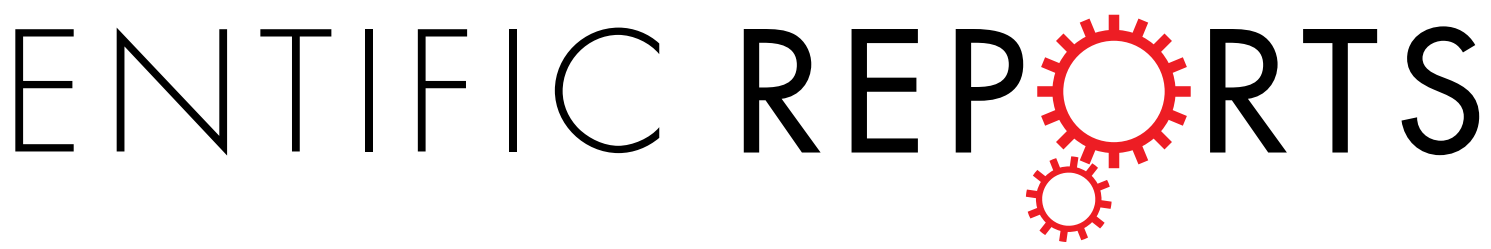

\title{
Heterologous expression of the yeast Tpo1p or Pdr5p membrane transporters in Arabidopsis confers plant xenobiotic tolerance
}

Estelle Remy ${ }^{1}$, María Niño-González $\mathbb{1}^{1}$, Cláudia P. Godinho $\mathbb{1}^{2}$, Tânia R. Cabrito², Miguel C. Teixeira $\mathbb{1}^{2}$, Isabel Sá-Correia ${ }^{2}{ }^{2} \&$ Paula Duque ${ }^{(1)}{ }^{1}$

Soil contamination is a major hindrance for plant growth and development. The lack of effective strategies to remove chemicals released into the environment has raised the need to increase plant resilience to soil pollutants. Here, we investigated the ability of two Saccharomyces cerevisiae plasmamembrane transporters, the Major Facilitator Superfamily (MFS) member Tpo1p and the ATP-Binding Cassette (ABC) protein Pdr5p, to confer Multiple Drug Resistance (MDR) in Arabidopsis thaliana. Transgenic plants expressing either of the yeast transporters were undistinguishable from the wild type under control conditions, but displayed tolerance when challenged with the herbicides 2,4-D and barban. Plants expressing SCTPO1 were also more resistant to the herbicides alachlor and metolachlor as well as to the fungicide mancozeb and the $\mathrm{Co}^{2+}, \mathrm{Cu}^{2+}, \mathrm{Ni}^{2+}, \mathrm{Al}^{3+}$ and $\mathrm{Cd}^{2+}$ cations, while ScPDR5expressing plants exhibited tolerance to cycloheximide. Yeast mutants lacking Tpo1p or Pdr5p showed increased sensitivity to most of the agents tested in plants. Our results demonstrate that the $S$. cerevisiae Tpo1p and Pdr5p transporters are able to mediate resistance to a broad range of compounds of agricultural interest in yeast as well as in Arabidopsis, underscoring their potential in future biotechnological applications.

Along decades, the development of human activities such as industry has led to the release of large amounts of toxic substances into the environment in the form of organic pollutants and heavy metals. Despite the urgent need to remove toxic substances from natural environments due to the risk they pose to wild life and to human health, current strategies to decontaminate soils are expensive and of low effectivity, normally involving the disposal of at least part of the soil in storage facilities ${ }^{1}$. Although some plant species are able to remove soil pollutants or at least to reduce their hazard, this ability having being exploited in a strategy called phytoremediation ${ }^{1}$, many of the plant species of economic importance (e.g. crops) are not naturally tolerant to the toxic effects of soil pollutants. The quest for strategies to make plants more resilient to such effects is therefore imperative.

The heterologous expression of proteins known to mediate resistance to xenobiotics in their native organisms in other, more sensitive, species has proved a successful means of enhancing plant resistance to toxic substances. For instance, the expression of OYE2, a dehydrogenase from Saccharomyces cerevisiae, in Arabidopsis thaliana led to an increased plant tolerance to 2,4,6-Trinitrotoluene (TNT) and a higher capacity of removing the compound from the media ${ }^{2}$. An engineered bacterial enzyme, dicamba monooxigenase (DMO), was shown to confer resistance to the herbicide dicamba in tobacco, tomato, Arabidopsis, and soybean plants ${ }^{3}$, thus representing a potential tool for weed resistance management in crops.

Among the types of proteins used for heterologous expression purposes, transporters have shown to be particularly suitable. Arabidopsis and tobacco plants expressing a wheat vacuolar $\mathrm{H}^{+}$-pyrophosphatase, TaVP1, were more tolerant to $\mathrm{Cd}^{2+}$ and accumulated more of the cation than their wild-type counterparts ${ }^{4,5}$. Expression of AtHMA4, a P1B-ATPase, or of its C-terminus in tobacco also modified $\mathrm{Cd}^{2+}$ as well as $\mathrm{Zn}^{2+}$ root/shoot partitioning and tolerance ${ }^{6}$. A similar effect was observed regarding arsenic resistance when the yeast proton gradient-driven antiporter ScACR3 gene was introduced into Arabidopsis ${ }^{7}$. In yet another example, two

${ }^{1}$ Instituto Gulbenkian de Ciência, 2780-156, Oeiras, Portugal. ' Institute for BioEngineering and Biosciences (iBB), Department of Bioengineering, Instituto Superior Técnico, Universidade de Lisboa, 1049-001, Lisbon, Portugal. Correspondence and requests for materials should be addressed to P.D. (email: duquep@igc.gulbenkian.pt) 
Multidrug Resistance-associated Proteins (MRPs) belonging to the ABC superfamily of transporters from S. cerevisiae, Ycflp and Vmrlp, conferred $\mathrm{Cd}^{2+}$ and $\mathrm{Pb}^{2+}$ resistance to transgenic poplar plants ${ }^{8}$.

The generation of transgenic plants bearing resistance to multiple xenobiotics can easily require several genetic modifications, and thus the identification of single genes conferring Multiple Drug Resistance (MDR) in plant systems is highly desirable. MDR is in most cases conferred by multidrug transporters of the ATP-Binding Cassette Superfamily (ABC) and Major Facilitator Superfamily (MFS). Among the ABC and MFS MDR transporters in the model eukaryote $S$. cerevisiae, the pleiotropic drug resistance protein $5(\mathrm{Pdr} 5 \mathrm{p})$ and the polyamine transporter 1 (Tpolp), respectively, stand out as the transporters conferring resistance to a wider array of drugs and xenobiotics ${ }^{9,10}$. Furthermore, both proteins confer specific resistance to agriculture-relevant stress agents. Tpolp, for example, is a determinant of yeast resistance to auxinic herbicides such as 2,4-dichlorophenoxyacetic acid $(2,4-\mathrm{D})^{11}$. Pdr1p, a transcription factor also determining 2,4-D resistance, is responsible for the activation of Tpolp expression upon sudden exposure to the herbicide ${ }^{11}$. Tpolp is also involved in the resistance to other chemical compounds, such as the herbicide barban or the fungicide mancozeb, and to toxic ions such as $\mathrm{Cd}^{2+}$ and $\mathrm{Al}^{3+12}$. Interestingly, the expression of an MFS member from Arabidopsis, AtZIFL1, in yeast also resulted in increased resistance to 2,4-D, and to the $\mathrm{Tl}^{3+}$ and $\mathrm{Al}^{3+}$ cations ${ }^{12}$. The $S$. cerevisiae Pdr5p transporter has also been reported to be both under Pdr1p transcriptional control upon exposure to 2,4-D and essential for the resistance to this herbicide ${ }^{11}$.

In the present study, we analysed the potential of the S. cerevisiae Tpolp and Pdr5p transporters to confer multidrug resistance in $A$. thaliana. Transgenic plants expressing either of these transporters were generated and then challenged with diverse chemical species to which Tpolp and/or Pdr5p mediate resistance in yeast. We found that heterologous expression of ScTPO1 or ScPDR5 in Arabidopsis resulted in enhanced plant tolerance to both the 2,4-D and barban herbicides and either to the fungicide mancozeb, the herbicides alachlor and metolachlor or to the fungicide cycloheximide, respectively. Moreover, resistance to toxic concentrations of several cations, namely $\mathrm{Co}^{2+}, \mathrm{Cu}^{2+}, \mathrm{Ni}^{2+}, \mathrm{Al}^{3+}$ and $\mathrm{Cd}^{2+}$, was observed in plants expressing ScTPO1. Thus, our results point to the yeast transporters Tpolp and Pdr5p as important tools to improve plant performance in xenobiotic-contaminated environments, with Tpolp showing to be particularly effective in mediating resistance against a broad range of toxic substances.

\section{Results \\ Heterologous expression of the yeast Tpo1p or Pdr5p transporters does not affect normal development of Arabidopsis plants. Previous work showed that Tpolp and Pdr5p are both involved in $S$. cerevisiae resistance to herbicides such as $2,4-\mathrm{D}^{11}$. To evaluate whether the expression of these yeast transport- ers would also be able to confer plant xenobiotic resistance, we transformed $A$. thaliana plants with the coding sequences of either the TPO1 or the PDR5 S. cerevisiae genes under the control of the strong constitutive 35S promoter. \\ All isolated Arabidopsis transgenic lines expressed the corresponding transcript (Fig. 1a, upper panels), with real-time RT-PCR revealing levels about 1000-fold higher for the ScPDR5 transgene than for ScTPO1, and still considerable differences in expression levels, though within the same order of magnitude, between lines express- ing the same yeast transporter, possibly due to differences in transgene copy number (Fig. 1a, lower panels). In general, the independent transgenic lines obtained showed wild-type behaviour during development under con- trol conditions (Fig. $1 \mathrm{~b}$ and Table 1), although the TPO1 transgenic lines L3 and L5 and the PDR5 transgenic line L2 were found to exhibit delayed growth when compared with wild-type plants (Fig. 1b). Indeed, seedlings cor- responding to these lines displayed slower elongation of the primary root (PR) than the wild type, but enhanced lateral root (LR) development, as measured through LR density and total length (Table 1). From a biotechnologi- cal point of view, the introduction of a transgene should confer (a) particular advantage(s) without negatively affecting other equally desirable traits and, for this reason, the TPO1 L3, TPO1 L5 and PDR5 L2 transgenic lines were discarded from further analyses.}

The yeast Tpo1p and Pdr5p transporters are also targeted to the plasma membrane in plant cells. We next analysed the subcellular localisation of the Tpolp and Pdr5p transporters in plant cells by means of translational fusions with yellow fluorescent protein (YFP) or green fluorescent protein (GFP) (Fig. 2). Both protoplasts isolated from Arabidopsis leaf mesophyll cells and whole Arabidopsis plants were transformed with either the transporter-YFP fusion or YFP alone driven by the 35S promoter. In parallel, Nicotiana tabacum leaves were co-transfected with either the 35S:ScTPO1-GFP or 35S:ScPDR5-GFP constructs and a plasma-membrane or a tonoplast marker.

As shown in Fig. 2a-f, upon both transient expression in isolated protoplasts and stable expression in transgenic plants, Tpolp localised to the plasma membrane of Arabidopsis cells. Accordingly, in N. tabacum leaves, the Tpolp-GFP fusion co-localised with the plasma-membrane marker (Fig. 2o-r) but not with the tonoplast marker (Fig. $2 \mathrm{~g}-\mathrm{j}$ ), confirming that, in planta like in yeast, the S. cerevisiae Tpolp transporter localises to the plasma membrane.

Although the fluorescence signal arising from the ScPDR5-YFP construct was not detectable in Arabidopsis cells, co-localisation of the Pdr5p-GFP fusion with the plasma-membrane (Fig. $2 \mathrm{~s}-\mathrm{v}$ ) but not with the tonoplast (Fig. $2 \mathrm{k}-\mathrm{n}$ ) marker was again observed in N. tabacum leaves, indicating that the yeast Pdr5p transporter also maintains its subcellular localisation when heterologously expressed in plant cells.

The yeast Tpo1p and Pdr5p transporters confer resistance to the 2,4-D herbicide in Arabidopsis. In S. cerevisiae, both the Tpolp and the Pdr5p transporters are known to be involved in the resistance to chlorinated phenoxyacetic acid herbicides such as $2,4-\mathrm{D}^{11}$. To investigate whether heterologous 
a
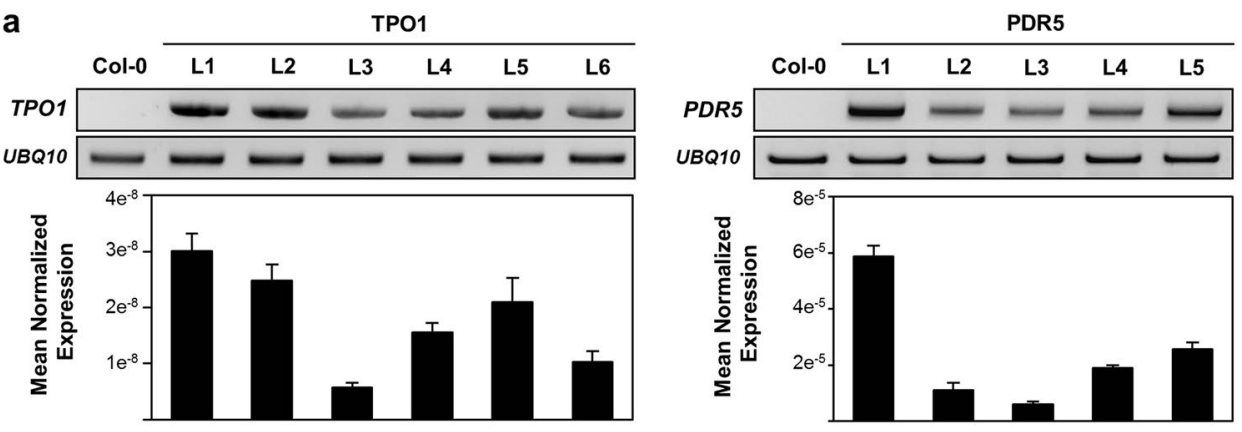

b
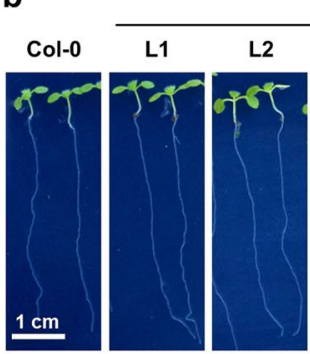

TPO1
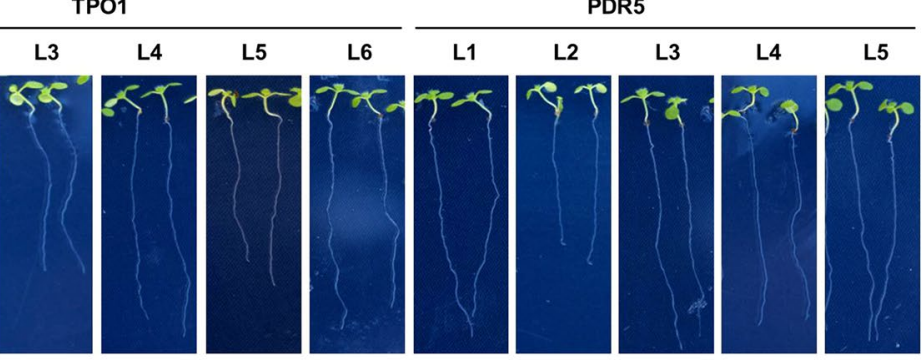

Figure 1. Transgene expression and phenotype under control conditions of transgenic Arabidopsis seedlings expressing the yeast Tpo1p or Pdr5p transporters. (a) Standard RT-PCR (upper panels) and real-time RTPCR (lower panels) analysis of S. cerevisiae TPO1 (left) and PDR5 (right) transcript levels in 7-d old seedlings of the wild type (Col-0) and TPO1 (TPO1L1-L6) or PDR5 (PDR5L1-L5) transgenic lines. Expression of the UBQ10 gene was used as a loading control or as a reference gene for standard RT-PCR and real-time RT-PCR, respectively. Values represent means $\pm \mathrm{SE}(n=4)$, with similar results being obtained in two independent experiments. (b) Representative images of 7-d old seedlings of the wild type (Col-0) and TPO1 (TPO1L1-L6) or PDR5 (PDR5L1-L5) transgenic lines grown under control conditions.

expression of the yeast ScTPO1 or ScPDR5 genes can confer plant tolerance to these compounds, we analysed the response of the Arabidopsis ScTPO1- and ScPDR5-expressing lines to a 2,4-D challenge (Fig. 3).

At the level of PR elongation, the TPO1 transgenic lines L1, L2 and L4 and the PDR5 transgenic lines L1 and L5 were clearly less affected by the inhibitory effects of the herbicide than the wild type and the other transgenic lines (Fig. 3a and b, upper panel). The increase in plant tolerance ranged from 15 to $23 \%$ after a $0.02 \mu \mathrm{M} 2,4-\mathrm{D}$ challenge and was even more pronounced at $0.04 \mu \mathrm{M} 2,4-\mathrm{D}$, with an increase between 20 and $57 \%$. Furthermore, a higher shoot biomass was detected in the ScTPO1-expressing lines L1 and L2 and in the ScPDR5-expressing line L1 (Fig. 3b, lower panel), with a rise in tolerance very similar to that observed for the roots $(15-23 \%$ at the lowest and $20-36 \%$ at the highest concentration of the herbicide). Importantly, the magnitude of the 2,4-D response largely correlated with the levels of heterologous expression of the corresponding yeast transporter in each transgenic line (see Fig. 1a). Despite the increased resistance to this auxinic herbicide, our results indicate that heterologous expression of ScTPO1 and ScPDR5 in Arabidopsis does not affect endogenous auxin metabolism, as PR elongation in transgenic plants treated with the native auxin indole-acetic acid (IAA) was similar to that of wild-type plants (Supplementary Fig. S1).

The mechanism by which Tpolp confers 2,4-D resistance in yeast is the extrusion of this compound from cells $^{12}$. Consistent with this, our measurements of ${ }^{14} \mathrm{C}-2,4-\mathrm{D}$ accumulation in roots were lower for the three TPO1 transgenic lines exhibiting 2,4-D resistance at the level of PR elongation than for wild-type plants (Fig. 3c). The two PDR5 lines whose PRs displayed reduced 2,4-D sensitivity also accumulated significantly less herbicide than the wild type (Fig. 3c), suggesting that 2,4-D resistance conferred by Pdr5p involves a mechanism similar to that proposed for Tpolp.

Tpo1p and Pdr5p confer tolerance to several other agricultural pesticides in yeast and Arabidopsis. In addition to 2,4-D, the yeast $\Delta t p o 1$ mutant displays decreased resistance to other compounds of agricultural interest, namely barban, a herbicide, and mancozeb, a fungicide ${ }^{12}$, both of which inhibit the growth of Arabidopsis plants (Supplementary Fig. S2a). To examine the possibility that the Tpo1p and Pdr5p transporters confer resistance to these pesticides also in plants, we challenged the Arabidopsis ScPDR5 and ScTPO1-expressing lines displaying the highest resistance to 2,4-D with either barban or mancozeb. With the exception of TPO1L4, PR elongation in the transgenic lines was less inhibited by barban than in the wild type, with increases in tolerance ranging between $17 \%$ and $40 \%$ or $44 \%$ and $79 \%$ for the lowest or highest barban concentration, respectively, although at the highest concentration tested the PDR5L5 line also behaved similarly to wild-type plants (Fig. 4). The fresh weight of transgenic shoots was also higher than that of wild-type shoots, showing increases between 24\% and 49\%, except for TPO1L4 and PDR5L5 (Fig. 4b, lower panel). As for mancozeb, the growth of 


\begin{tabular}{|c|c|c|c|c|}
\hline Genotypes & FW (mg per plant) & PRE (cm) & $\operatorname{LRD}(\mathrm{LR} / \mathrm{cm})$ & TLRL $(\mathrm{cm})$ \\
\hline Col-0 & $25.59 \pm 2.62$ & $4.27 \pm 0.48$ & $1.47 \pm 0.32$ & $5.44 \pm 1.15$ \\
\hline \multicolumn{5}{|c|}{ ScTPO1-expressing } \\
\hline TPO1L1 & $\begin{array}{l}26.57 \pm 3.38 \\
(0.277571)\end{array}$ & $\begin{array}{l}4.52 \pm 0.30 \\
(0.059858)\end{array}$ & $\begin{array}{l}1.37 \pm 0.32 \\
(0.220766)\end{array}$ & $\begin{array}{l}6.03 \pm 1.30 \\
(0.188536)\end{array}$ \\
\hline TPO1L2 & $\begin{array}{l}27.33 \pm 4.18 \\
(0.183777)\end{array}$ & $\begin{array}{l}4.16 \pm 0.28 \\
(0.232608)\end{array}$ & $\begin{array}{l}1.62 \pm 0.28 \\
(0.099624)\end{array}$ & $\begin{array}{l}5.43 \pm 0.66 \\
(0.493820)\end{array}$ \\
\hline TPO1L3 & $\begin{array}{l}21.40 \pm 5.12 \\
(0.037274)\end{array}$ & $\begin{array}{l}2.99 \pm 0.62 \\
\left(1.511 \mathrm{e}^{-05}\right)\end{array}$ & $\begin{array}{l}2.80 \pm 0.99 \\
(0.000142)\end{array}$ & $\begin{array}{l}7.46 \pm 2.13 \\
(0.000142)\end{array}$ \\
\hline TPO1L4 & $\begin{array}{l}24.73 \pm 2.04 \\
(0.253392)\end{array}$ & $\begin{array}{l}4.13 \pm 0.38 \\
(0.202709)\end{array}$ & $\begin{array}{l}1.52 \pm 0.38 \\
(0.350817)\end{array}$ & $\begin{array}{l}5.72 \pm 0.91 \\
(0.308970)\end{array}$ \\
\hline TPO1L5 & $\begin{array}{l}21.58 \pm 3.69 \\
(0.017224)\end{array}$ & $\begin{array}{l}2.98 \pm 0.82 \\
\left(9.023 \mathrm{e}^{-05}\right)\end{array}$ & $\begin{array}{l}2.09 \pm 0.66 \\
(0.006238)\end{array}$ & $\begin{array}{l}6.97 \pm 2.23 \\
(0.006238)\end{array}$ \\
\hline TPO1L6 & $\begin{array}{l}26.98 \pm 5.17 \\
(0.268335)\end{array}$ & $\begin{array}{l}4.33 \pm 0.46 \\
(0.373284)\end{array}$ & $\begin{array}{l}1.48 \pm 0.37 \\
(0.455183)\end{array}$ & $\begin{array}{l}5.12 \pm 1.32 \\
(0.455183)\end{array}$ \\
\hline \multicolumn{5}{|c|}{ ScPDR5-expressing } \\
\hline PDR5L1 & $\begin{array}{l}26.46 \pm 3.97 \\
(0.318385)\end{array}$ & $\begin{array}{l}4.30 \pm 0.46 \\
(0.435980)\end{array}$ & $\begin{array}{l}1.80 \pm 0.76 \\
(0.075609)\end{array}$ & $\begin{array}{l}6.05 \pm 0.98 \\
(0.149458)\end{array}$ \\
\hline PDR5L2 & $\begin{array}{l}22.26 \pm 3.83 \\
(0.039194)\end{array}$ & $\begin{array}{l}2.89 \pm 0.68 \\
\left(1.230 \mathrm{e}^{-05}\right)\end{array}$ & $\begin{array}{l}2.56 \pm 0.77 \\
(0.000119) \\
\end{array}$ & $\begin{array}{l}9.88 \pm 2.15 \\
(0.000135)\end{array}$ \\
\hline PDR5L3 & $\begin{array}{l}26.46 \pm 4.57 \\
(0.334319)\end{array}$ & $\begin{array}{l}4.48 \pm 0.24 \\
(0.088359)\end{array}$ & $\begin{array}{l}1.34 \pm 0.69 \\
(0.267862)\end{array}$ & $\begin{array}{l}5.29 \pm 1.29 \\
(0.411617) \\
\end{array}$ \\
\hline PDR5L4 & $\begin{array}{l}26.38 \pm 2.26 \\
(0.279327)\end{array}$ & $\begin{array}{l}4.17 \pm 0.30 \\
(0.271002)\end{array}$ & $\begin{array}{l}1.58 \pm 0.28 \\
(0.162667)\end{array}$ & $\begin{array}{l}5.60 \pm 0.85 \\
(0.382948)\end{array}$ \\
\hline PDR5L5 & $\begin{array}{l}25.01 \pm 4.78 \\
(0.391651)\end{array}$ & $\begin{array}{l}4.26 \pm 0.42 \\
(0.468568)\end{array}$ & $\begin{array}{l}1.50 \pm 0.11 \\
(0.357897)\end{array}$ & $\begin{array}{l}5.03 \pm 0.64 \\
(0.212272)\end{array}$ \\
\hline
\end{tabular}

Table 1. Growth parameters under control conditions of transgenic Arabidopsis lines expressing the yeast Tpolp or Pdr5p transporters. Shoot biomass (fresh weight, FW), primary root elongation (PRE), lateral root density (LRD) and total lateral root length (TLRL) of 7-d (PRE) or 14-d (FW, LRD, TLRL) old seedlings from the wild type (Col-0) or ScTPO1- and ScPDR5-expressing lines grown in control medium. Values represent means $\pm \mathrm{SD}(n=8)$, with similar results being obtained in three independent experiments performed with different seed batches. Numbers in parentheses indicate the $P$ value (comparison with the wild type) obtained by Student's $t$-test.

ScTPO1-expressing plants was less sensitive to the effects of the fungicide than that of the ScPDR5 transgenic lines or wild-type plants (Fig. 4) although for TPO1L4, the plant line expressing lower amounts of ScTPO1 transcript (see Fig. 1a), no alterations in mancozeb sensitivity were observed at the highest concentration (Fig. 4b). Although in general ScTPO1 transgenic plants resisted better to barban than to mancozeb, the root and shoot tolerance to the latter compound still increased by $17-48 \%$ and $8-19 \%$, respectively. These results prompted us to investigate the response of the yeast $\Delta p d r 5$ mutant to barban and mancozeb. In line with what was observed in planta, yeast cells lacking Pdr5p were more sensitive to barban than the wild-type, whereas the wild-type and the mutant strain were equally sensitive to mancozeb (Supplementary Fig. S3). In the mancozeb experiment, we included the $\Delta t p o 1$ strain as a control, given that this mutant has been previously reported to display hypersensitivity to the fungicide ${ }^{12}$.

Encouraged by our finding that the multidrug resistance conferred by two yeast plasma-membrane transporters also functions in Arabidopsis, and owing to the decreased resistance of S. cerevisiae PDR5 mutants to cycloheximide $^{13}$, we next analysed the effects of Pdr5p expression on plant responses to this compound, which has been used in agriculture as a large-spectrum fungicide under the name actidione. Both the PDR5L1 and PDR5L5 transgenic lines were tested at two different concentrations of cycloheximide, exhibiting a longer PR ( $\sim 27 \%$ increase) and higher fresh shoot weight than wild-type plants (Fig. 5). The effect on shoot weight was subtle ( 11\% increase) and only felt at the highest concentration tested (Fig. 5b, lower panel), most likely because this parameter is less affected by cycloheximide than elongation of the PR (Supplementary Fig. S2b).

We also investigated the in planta role of Tpolp in the resistance to other pesticides not yet reported in yeast, such as alachlor and metolachlor. Both these herbicides markedly impaired Arabidopsis growth even at very low concentrations (Supplementary Fig. S2c). As seen in Fig. 6, both PR elongation and shoot biomass of two independent plant lines expressing ScTPO1 (TPO1L1 and TPO1L2) were less affected by alachlor and metolachlor than wild-type plants, although at the higher concentrations tested TPO1L2 shoot biomass was similar to that of the wild type (Fig. 6b, lower panel). The ScTPO1 transgenic lines exhibited a moderate increase in the tolerance to both herbicides, ranging from 23 to $34 \%$ or 17 to $29 \%$ and 21 to $46 \%$ or 16 to $33 \%$ for alachlor and metolachlor at the PR elongation or shoot biomass levels, respectively. Tpolp-mediated resistance to both alachlor and metolachlor was also observed in yeast, with the $\Delta t p o 1$ mutant strain showing enhanced sensitivity to these compounds relative to the wild type when grown in either solid (Supplementary Fig. S4a) or liquid (Supplementary Fig. S4b) media. 

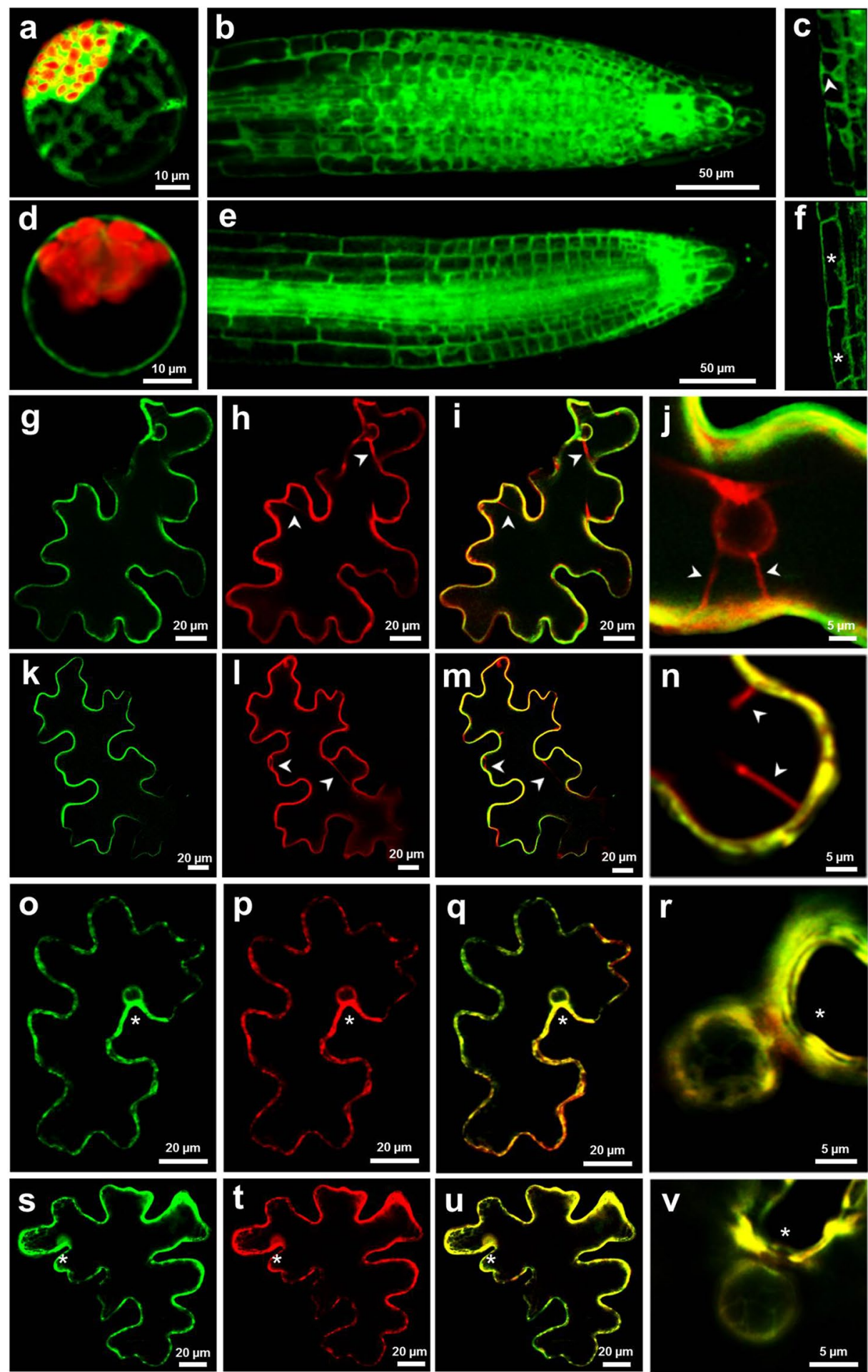

Figure 2. Subcellular localisation of the yeast Tpolp and Pdr5p transporters in plant cells. (a-f) Confocal laser scanning microscopy images of Arabidopsis mesophyll protoplasts (a,d) or transgenic root tips (b,c,e,f), transiently or stably expressing either YFP alone (a,b,c) or the ScTPO1-YFP fusion (d,e,f), respectively, under the control of the $35 \mathrm{~S}$ promoter. Root epidermal cell close-ups (c,f) are shown. The YFP and chloroplast autofluorescence signals are visualized by green and red coloration, respectively. (g-v) Confocal laser scanning microscopy images of individual tobacco leaf epidermal cells transiently expressing the ScTPO1-

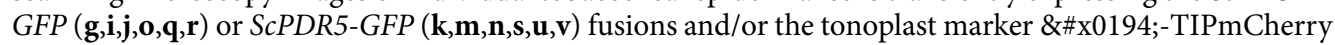
$(\mathbf{h}, \mathbf{i}, \mathbf{j}, \mathbf{l}, \mathbf{m}, \mathbf{n})$ or the plasma-membrane marker PIP2A-mCherry $(\mathbf{p}, \mathbf{q}, \mathbf{r}, \mathbf{t}, \mathbf{u}, \mathbf{v})$ under the control of the $35 \mathrm{~S}$ promoter. Merged images of whole-cell views $(\mathbf{i}, \mathbf{m}, \mathbf{q}, \mathbf{u})$ or nucleus close-ups $(\mathbf{j}, \mathbf{n}, \mathbf{r}, \mathbf{v})$ are shown. Arrowheads point to transvacuolar strands, and asterisks indicate fluorescence signals approaching the nucleus only on the side facing the exterior of the cell (o-v) or surrounding the nucleus (f). The GFP and mCherry signals are visualized by green and red coloration, respectively. 
a

TPO1
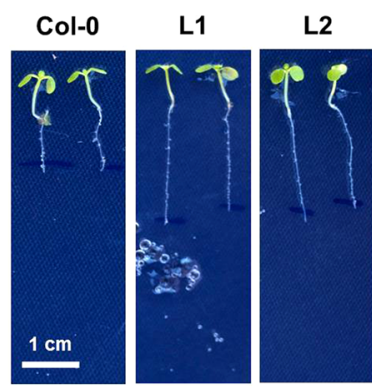

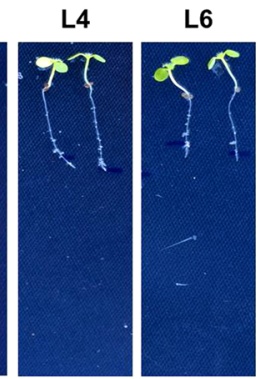

$+0.04 \mu M$ 2,4-D

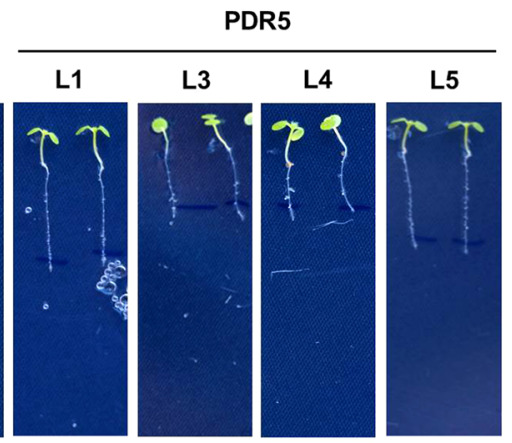

C b

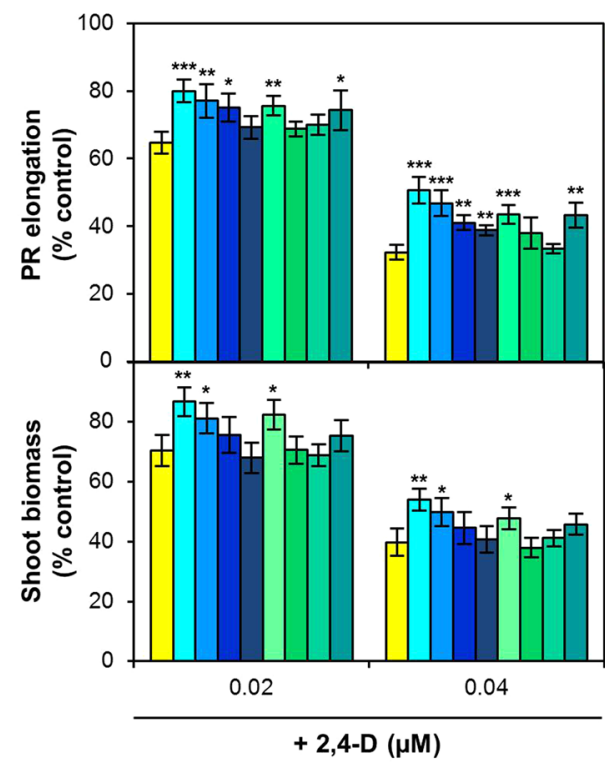

口Col-0 口TP01L1 口TP01L2 -TP01L4 -TP01L6 口PDR5L1 口PDR5L3 $\square$ PDR5L4 口PDR5L5

Figure 3. Effects of a 2,4-D challenge on transgenic Arabidopsis lines expressing the yeast Tpolp or Pdr5p transporters. (a) Representative images of 7-d old seedlings of the wild type (Col-0) and TPO1 (TPO1L1, L2, L4, L6) or PDR5 (PDR5L1, L3, L4, L5) transgenic lines grown on medium containing 2,4-D. Plant growth under control conditions is shown in Fig. 1 and Table 1. (b) Effect of 2,4-D toxicity on PR elongation (upper panel) and shoot biomass (lower panel) of seedlings of the wild type (Col-0) and TPO1 (TPO1L1, L2, L4, L6) or PDR5 (PDR5L1, L3, L4, L5) transgenic lines. (c) Accumulation of ${ }^{14} \mathrm{C}-2,4-\mathrm{D}$ in root tips from 5-d old seedlings of the wild type (Col-0) and TPO1 (TPO1L1, L2, L4) or PDR5 (PDR5L1, L5) transgenic lines. Values represent means $\pm \mathrm{SD}(n=8)$, with similar results being obtained in three independent experiments performed with different seed batches. Asterisks denote statistically significant differences between ScTPO1- or ScPDR5expressing lines and the wild type $(* P<0.05, * * P<0.01, * * * P<0.001$; Student's $t$-test $)$.

Tpo1p confers plant tolerance to rhizotoxic cations. Tpolp has been previously reported to be involved in the resistance to some metal cations, such as $\mathrm{Al}^{3+}$ or $\mathrm{Cd}^{2+}$, in yeast ${ }^{12}$. In plants, transition metals like $\mathrm{Co}^{2+}, \mathrm{Cu}^{2+}$ or $\mathrm{Ni}^{2+}$ act as micronutrients, but become toxic at high concentrations.

To investigate the ability of the yeast Tpolp transporter to confer resistance to metal cations in plants, we analysed the growth of Arabidopsis ScTPO1-expressing lines upon exposure to two different concentrations of the $\mathrm{Co}^{2+}, \mathrm{Cu}^{2+}, \mathrm{Ni}^{2+}, \mathrm{Al}^{3+}$ or $\mathrm{Cd}^{2+}$ ions, selected based on our previously reported physiological analyses ${ }^{14}$. From Fig. 7 it is clear that transgenic lines TPO1L1 and TPO1L2 exhibited a longer PR than wild-type plants when grown in the presence of at least one concentration of each of the five cations, while TPO1L4 showed enhanced resistance only when treated with the lowest concentration of $\mathrm{Co}^{2+}$ or the highest concentration of $\mathrm{Ni}^{2+}\left(\mathrm{Fig}^{2} \mathrm{7a}\right.$ and $\mathrm{b}$, upper panel). The rise in plant tolerance was between $42 \%$, at the maximum $\mathrm{Co}^{2+}$ concentration, and $13 \%$, at the lowest $\mathrm{Cu}^{2+}$ concentration. Similar results were observed when fresh shoot weight was measured (Fig. 7b, lower panel), with tolerance to the cations being enhanced $11-45 \%$, although in the $\mathrm{Cu}^{2+}$ treatments only TPO1L1 showed to be more resistant than the wild type. None of the TPO1 transgenic lines displayed a phenotype when challenged with the highest concentration of $\mathrm{Cd}^{2+}$, probably because this level of toxicity is high enough to surpass Tpolp-mediated resistance. 
a

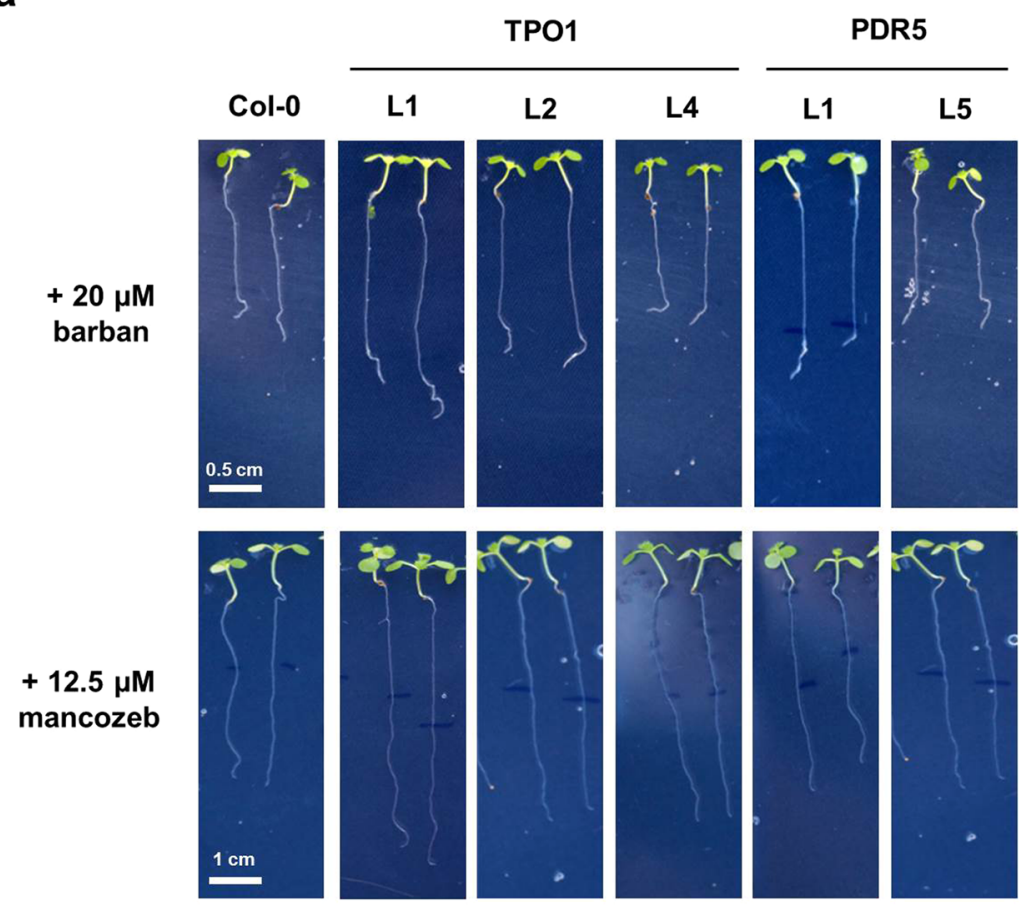

b
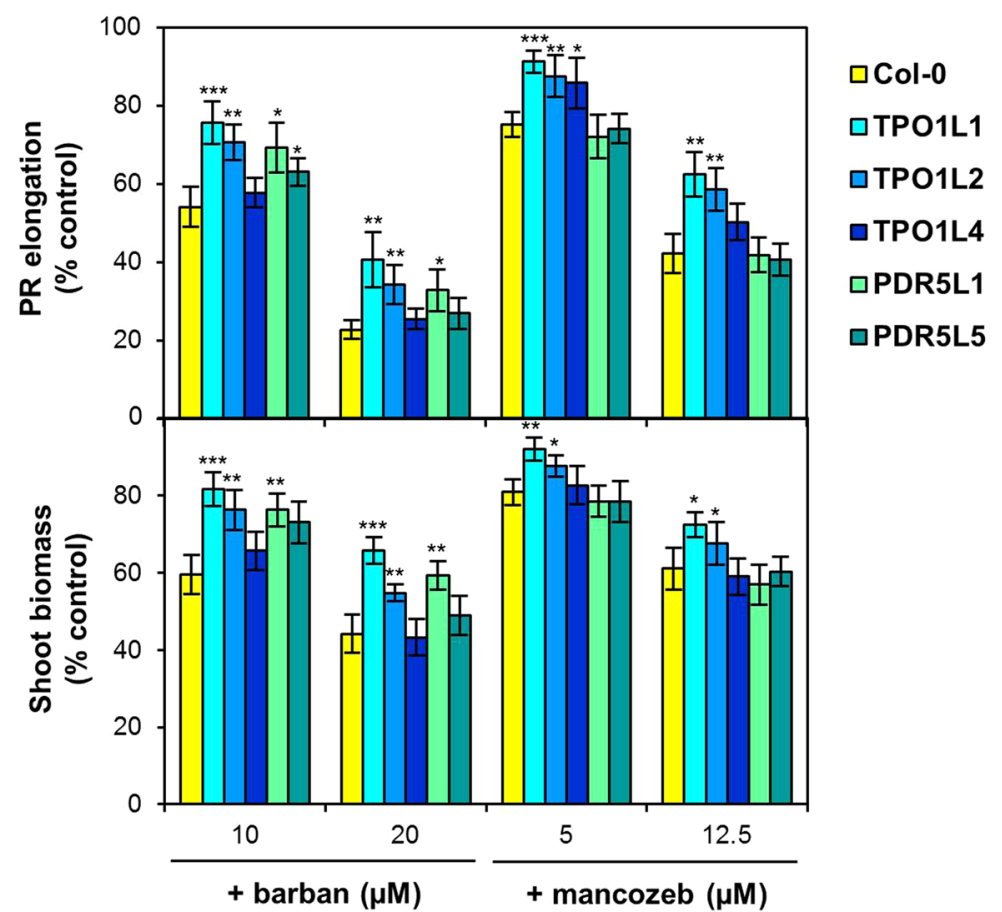

Figure 4. Effects of a barban or mancozeb challenge on transgenic Arabidopsis lines expressing the yeast Tpolp or Pdr5p transporters. (a) Representative images of 7-d old seedlings of the wild type (Col-0) and TPO1 (TPO1L1, L2, L4) or PDR5 (PDR5L1, L5) transgenic lines grown on media containing barban (upper panel) or mancozeb (lower panel). Plant growth under control conditions is shown in Fig. 1 and Table 1. (b) Effect of barban or mancozeb toxicity on PR elongation (upper panel) and shoot biomass (lower panel) of seedlings of the wild type (Col-0) and TPO1 (TPO1L1, L2, L4) or PDR5 (PDR5L1, L5) transgenic lines. Values represent means $\pm S D(n=8)$, with similar results being obtained in three independent experiments performed with different seed batches. Asterisks denote statistically significant differences between ScTPO1- or ScPDR5expressing lines and the wild type $(* P<0.05$, $* * P<0.01$, *** $P<0.001$; Student's $t$-test $)$. 
a

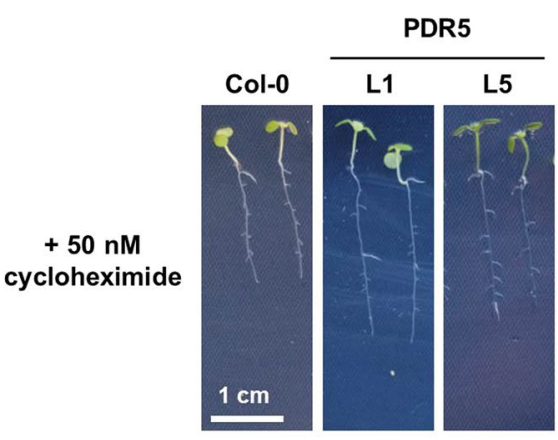

b

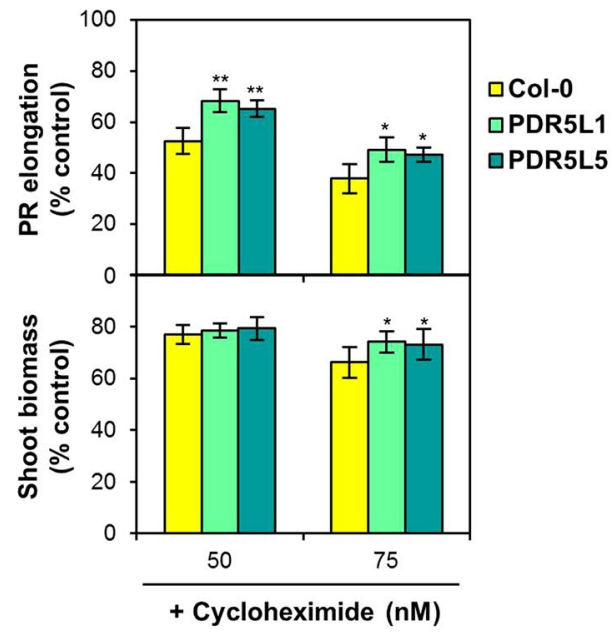

Figure 5. Effects of a cycloheximide challenge on transgenic Arabidopsis lines expressing the yeast Pdr5p transporter. (a) Representative images of 7-d old seedlings of the wild type (Col-0) and PDR5 (PDR5L1, L5) transgenic lines grown on medium containing cycloheximide. Plant growth under control conditions is shown in Fig. 1 and Table 1. (b) Effect of cycloheximide toxicity on PR elongation (upper panel) and shoot biomass (lower panel) of seedlings of the wild type (Col-0) and PDR5 (PDR5L1, L5) transgenic lines. Values represent means $\pm \mathrm{SD}(n=8)$, with similar results being obtained in three independent experiments performed with different seed batches. Asterisks denote statistically significant differences between ScPDR5-expressing lines and the wild type $(* P<0.05, * * P<0.01$; Student's $t$-test $)$.

We also checked whether $\mathrm{Al}^{3+}, \mathrm{Cd}^{2+}, \mathrm{Co}^{2+}, \mathrm{Cu}^{2+}$ and $\mathrm{Ni}^{2+}$ accumulation would be affected in ScTPO1-expressing plants upon challenge with each of these cations by comparing their respective content in transgenic and wild-type seedlings. Overall, no significant differences were observed between the plant line displaying the strongest transgene expression, TPO1L1, and the wild type, except for $\mathrm{Al}^{3+}$-treated plants, where the content of this cation was found to be higher in the transgenic line (Table 2).

Despite the increased tolerance to all five examined cations conferred by heterologous expression of the yeast Tpolp transporter in Arabidopsis, and in contrast to what has been reported for $\mathrm{Al}^{3+}$ and $\mathrm{Cd}^{2+}$, we were unable to detect a differential response between the yeast $\Delta t p o 1$ mutant and the wild-type strain to the transition metals $\mathrm{Co}^{2+}, \mathrm{Cu}^{2+}$ or $\mathrm{Ni}^{2+}$ (Supplementary Fig. S5).

\section{Discussion}

Soil contamination is a major hindrance to plant growth and productivity, and hence the development of effective strategies to overcome the toxic effects of soil pollutants is widely recognized as crucial. Here, we report the effects of transgenic expression of two S. cerevisiae plasma-membrane transporters, Tpolp, belonging to the MFS, and Pdr5p, an ABC superfamily member, on tolerance of the model plant $A$. thaliana towards several toxic substances, including agricultural pesticides and heavy metals.

Under control conditions, heterologous expression of the yeast ScTPO1 and ScPDR5 genes in Arabidopsis did not affect the growth of the transgenic lines generated, with the exception of two ScTPO1- and one ScPDR5-expressing lines. In fact, transgene expression levels in these three lines were comparable to those in the remaining transgenic plants, suggesting that the observed delay in growth is not related to heterologous expression of the yeast transporters, but rather to the regions of the Arabidopsis genome disrupted by random insertion 
a

TPO1

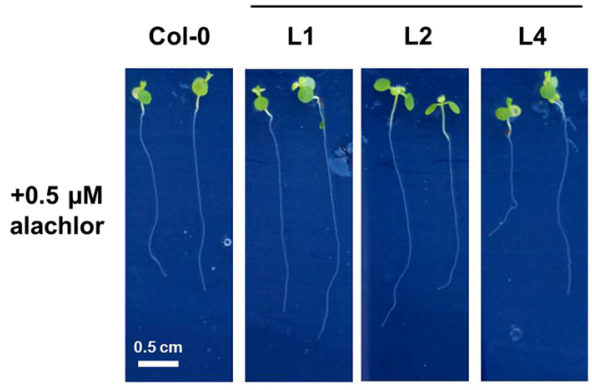

TPO1

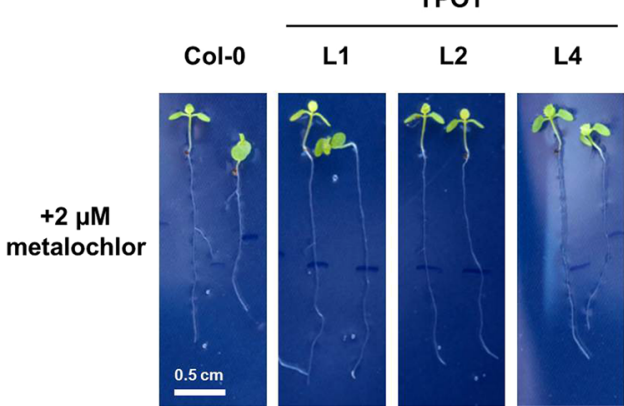

b

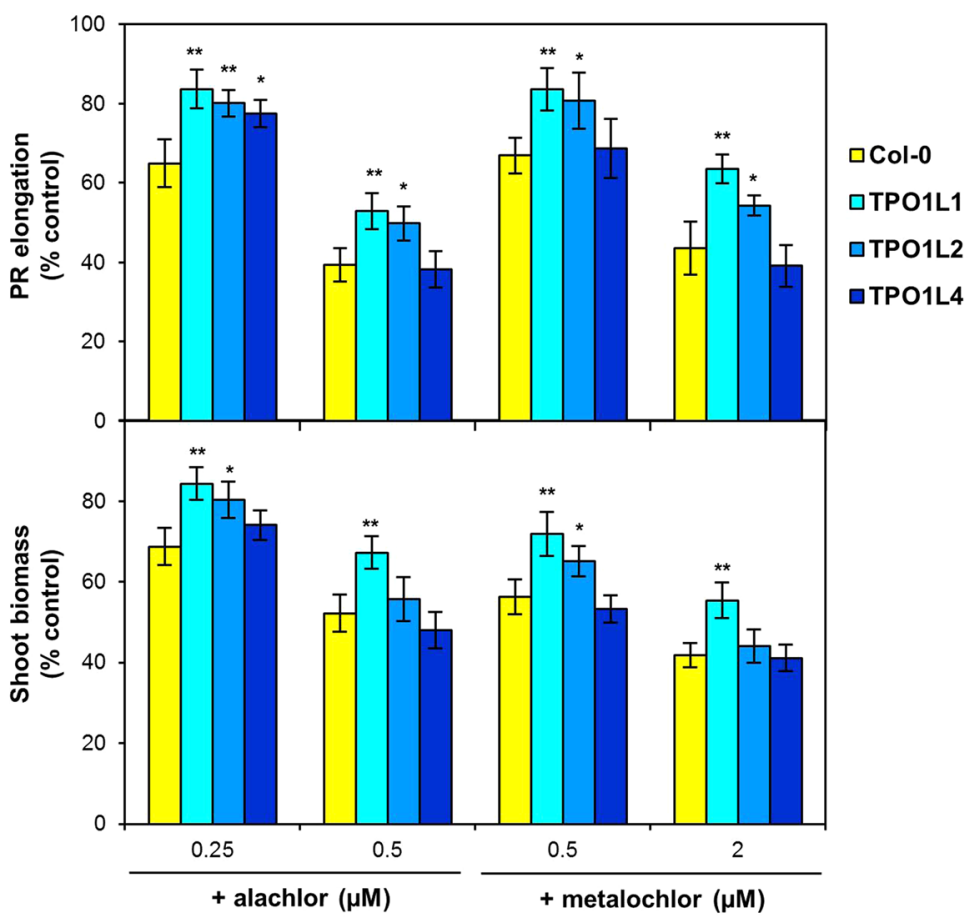

Figure 6. Effects of an alachlor or metalochlor challenge on transgenic Arabidopsis lines expressing the yeast Tpo1p transporter. (a) Representative images of 7-d old seedlings of the wild type (Col-0) and TPO1 (TPO1L1, L2, L4) transgenic lines grown on media containing alachlor (left panel) or metalochlor (right panel). Plant growth under control conditions is shown in Fig. 1 and Table 1. (b) Effect of alachlor or metalochlor toxicity on PR elongation (upper panel) and shoot biomass (lower panel) of seedlings of the wild type (Col-0) and TPO1 (TPO1L1, L2, L4) transgenic lines. Values represent means \pm SD $(n=8)$, with similar results being obtained in three independent experiments performed with different seed batches. Asterisks denote statistically significant differences between $S c T P O 1$-expressing lines and the wild type $(* P<0.05$, $* * P<0.01$; Student's $t$-test).

of the transgenes. Interestingly, expression of $S c P D R 5$ was always about three orders of magnitude higher than that of ScTPO1, despite the fact that both genes are being driven in planta by the same strong constitutive promoter, 35S. This discrepancy could be due to differential mRNA processing or stability and/or regulation of the transgenes at the transcription/translation levels ${ }^{15}$.

Globally, heterologous expression of the Tpo1p and Pdr5p yeast transporters in Arabidopsis conferred a moderate though significant increase in plant tolerance to a range of compounds. Generally, it might well be preferable for the plant to exhibit moderate resistance to several agents than to greatly resist a few. Still, enhancing the intensity of the observed broad tolerance could be highly desirable. One way to improve the transgenes' efficiency would be to target them to a specific site in the plant genome, thus selecting the most convenient location for the insertion and avoiding collateral effects, such as the disruption of genes crucial to plant survival. Endonuclease-based technologies such as Clustered Regularly Interspaced Short Palindromic Repeats (CRISPR)-Associated (Cas) ${ }^{16}$, Zinc-Finger Nucleases (ZFNs) ${ }^{17}$ or Transcription Activator-Like Effector Nucleases (TALENs) ${ }^{18}$ may be used for this purpose. Also, depending on the plant species in which the transgene is inserted, other promoters that are either more efficient or whose activity is specific to a tissue or a developmental stage could be employed when desirable. 
a

\begin{tabular}{llll} 
& \multicolumn{3}{c}{ TPO1 } \\
\cline { 2 - 4 } Col-0 & L1 & L2 & L4
\end{tabular}
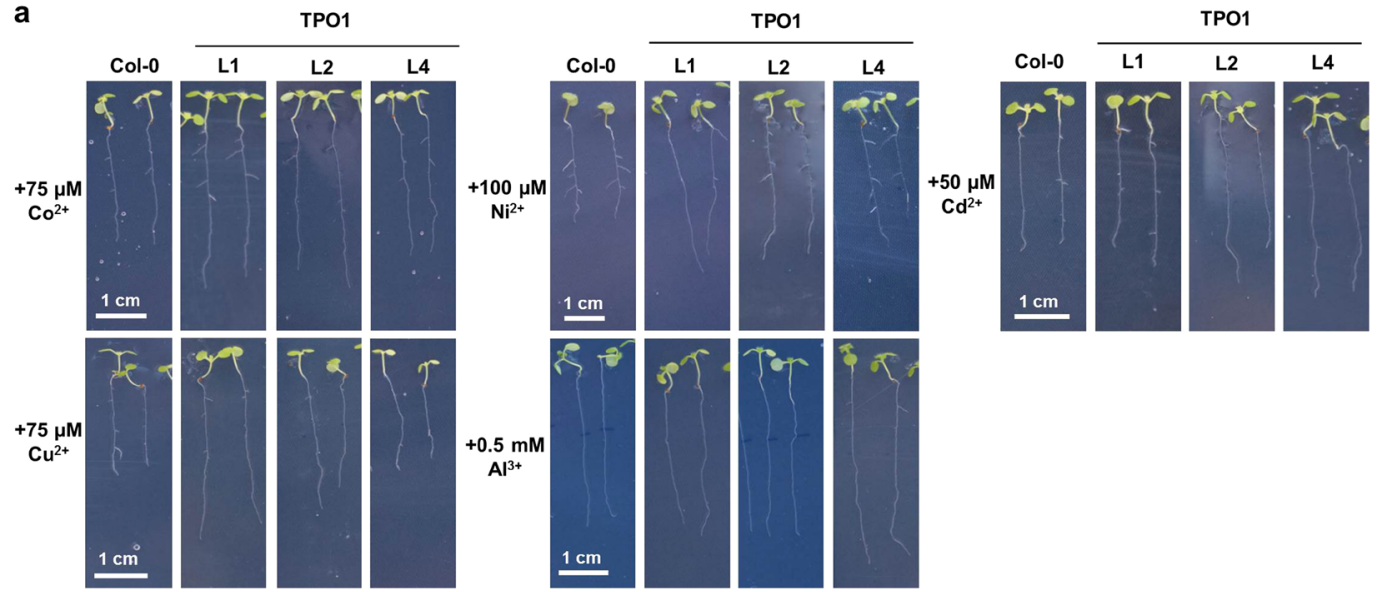

$1 \mathrm{~cm}$

b

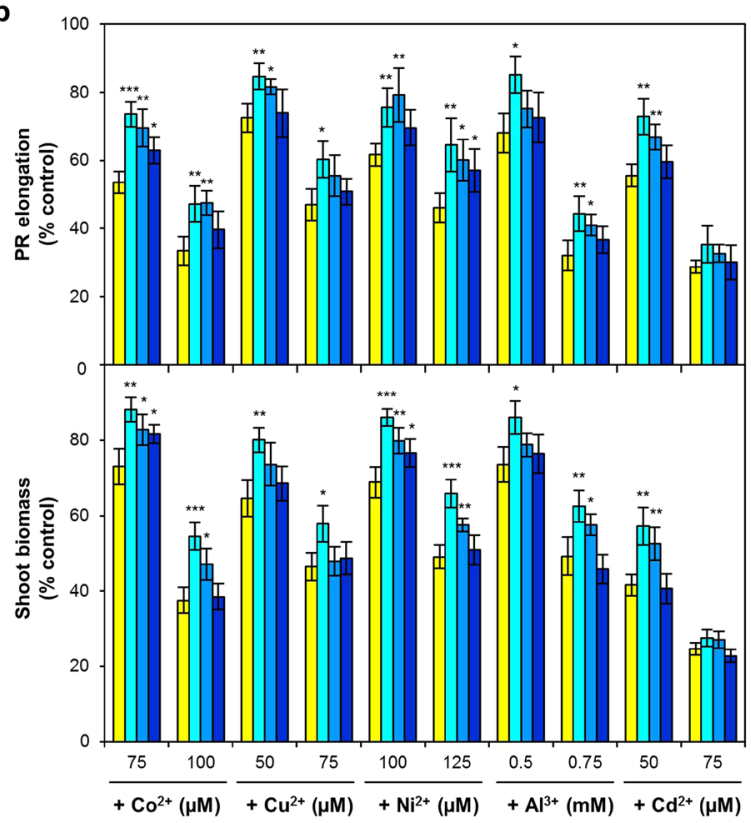

口Col-0

口TP01L1

口TPO1L2

ITPO1L4

Figure 7. Effects of rhizotoxic ion challenge on transgenic Arabidopsis lines expressing the yeast Tpolp transporter. (a) Representative images of 7-d old seedlings of the wild type (Col-0) and TPO1 (TPO1L1, L2, L4) transgenic lines grown on media containing $\mathrm{Co}^{2+}, \mathrm{Cu}^{2+}, \mathrm{Ni}^{2+}, \mathrm{Al}^{3+}$ or $\mathrm{Cd}^{2+}$. Plant growth under control conditions is shown in Fig. 1 and Table 1. (b) Effect of $\mathrm{Co}^{2+}, \mathrm{Cu}^{2+}, \mathrm{Ni}^{2+}, \mathrm{Al}^{3+}$ or $\mathrm{Cd}^{2+}$ toxicity on PR elongation (upper panel) and shoot biomass (lower panel) of seedlings of the wild type (Col-0) and TPO1 (TPO1L1, L2, L4) transgenic lines. Values represent means $\pm \mathrm{SD}(n=8)$, with similar results being obtained in three independent experiments performed with different seed batches. Asterisks denote statistically significant differences between ScTPO1-expressing lines and the wild type $(* P<0.05$, **P $P 0.01, * * * P<0.001$; Student's $t$-test).

\begin{tabular}{|l|l|l|}
\hline & Col-0 & TPO1L1 \\
\hline $\mathrm{Co}^{2+}$ & $298.36 \pm 43.78$ & $\begin{array}{l}306.48 \pm 22.42 \\
(0.742)\end{array}$ \\
\hline $\mathrm{Cu}^{2+}$ & $77.84 \pm 11.11$ & $\begin{array}{l}81.42 \pm 22.16 \\
(0.782)\end{array}$ \\
\hline $\mathrm{Ni}^{2+}$ & $186.38 \pm 19.78$ & $\begin{array}{l}192.97 \pm 94.67 \\
(0.896)\end{array}$ \\
\hline $\mathrm{Cd}^{2+}$ & $311.36 \pm 15.16$ & $\begin{array}{l}285.70 \pm 63.03 \\
(0.459)\end{array}$ \\
\hline $\mathrm{Al}^{3+}$ & $148.30 \pm 20.89$ & $\begin{array}{l}181.44 \pm 13.34 \\
(0.037)\end{array}$ \\
\hline
\end{tabular}

Table 2. Elemental analysis of a transgenic Arabidopsis line expressing the yeast Tpolp transporter. Total cation concentration ( $\mu \mathrm{g} \mathrm{g}^{-1} \mathrm{DW}$ ) of wild-type and transgenic ScTPO1-expressing Arabidopsis plants challenged with $0.5 \mathrm{mM} \mathrm{Al}^{3+}, 50 \mu \mathrm{M} \mathrm{Cd}^{2+}, 75 \mu \mathrm{M} \mathrm{Co}^{2+}, 50 \mu \mathrm{M} \mathrm{Cu}^{2+}$ and $100 \mu \mathrm{M} \mathrm{Ni}^{2+}$ (means $\left.\pm \mathrm{SD}, n=4\right)$. Numbers in parentheses indicate the $P$ value (comparison with the wild type) obtained by Student's $t$-test. 
In yeast, the ScTPO1 and ScPDR5 transporter genes are activated by Pdr1p, a transcriptional regulator determining yeast resistance to two widely used herbicides, 2-methyl-4-chlorophenoxyacetic acid (MCPA) and 2,4- $\mathrm{D}^{11}$. In addition, Tpolp has been shown to confer yeast resistance to the herbicide barban, the plant hormone IAA, the fungicide mancozeb, and the metal cations $\mathrm{Cd}^{2+}$ and $\mathrm{Al}^{3+12}$, whereas Pdr5p has been implicated in the resistance to a broad range of substances such as mycotoxins ${ }^{19}$, steroid hormones ${ }^{20}$, cycloheximide ${ }^{21}$, and a vast number of antifungal compound $\mathrm{s}^{22}$. Two possible explanations for the pleiotropic resistance promoted by Tpolp and Pdr5p can be envisaged. On the one hand, both these transporters appear to be important for cellular processes other than multidrug resistance, and therefore resistance to xenobiotics could represent a collateral effect of the transporter's function. For instance, Tpolp was postulated to mediate cell extrusion of polyamines to regulate their intracellular concentration $^{23,24}$. Polyamines are small aliphatic cations critical to growth and development of both eukaryotes and prokaryotes ${ }^{25}$, and hence their regulation is pivotal to cell fitness. Also, Pdr5p is involved in ATP-dependent phospholipid translocation ${ }^{26}$ and could thus modulate the permeability of the plasma membrane by altering its lipid composition. On the other hand, at least in some instances, multidrug resistance may be conferred directly by Tpo 1 and Pdr5. Indeed, many of the substances these transporters mediate resistance to are weak acid compounds, and it could well be that their dissociated counter-ions are Tpolp and/or Pdr5p substrates ${ }^{27}$.

ScTPO1-expressing plants challenged with cations accumulated levels of $\mathrm{Cd}^{2+}, \mathrm{Co}^{2+}, \mathrm{Cu}^{2+}$ and $\mathrm{Ni}^{2+}$ similar to the wild type and higher levels of $\mathrm{Al}^{3+}$, which would suggest that the Tpolp-mediated resistance mechanism does not involve extrusion of the cations from plant tissues. However, a direct mechanism of resistance cannot be excluded as the Tpolp transporter could act at the level of organ partitioning of these cations, reducing the magnitude of their negative effects on plant growth through their plant repartition. The fact that the transgenic plants accumulated more $\mathrm{Al}^{3+}$ than the wild type but were still less affected by this cation, whose first symptom of toxicity is inhibition of root growth ${ }^{28}$, could support this hypothesis. Taken together, the findings that Tpolp and Pdr5p are targeted to the same cellular membrane in yeast and transgenic Arabidopsis lines, that the biological processes in which these transporters have been implicated are conserved between plants and yeast, and that the resistance phenotypes conferred in transgenic plants are often reciprocal to those displayed by yeast loss-of-function mutants, strongly suggest that the general mechanisms by which xenobiotic resistance is achieved in Arabidopsis are the same as in yeast.

The weak acid 2,4-D is one of the ten most commonly used active pesticide ingredients in the U.S. ${ }^{29}$, and it is also extensively employed in agriculture worldwide. The importance of developing crops resistant to herbicidal compounds lies on the potential harmful effects of these substances on the crops they are protecting from weeds. Although varieties of some crops, such as cotton, corn and soybean, displaying resistance to 2,4-D (as well as to the glyphosate and glufosinate herbicides) have been developed and recently launched into the market ${ }^{30}$, weed resistance remains a major concern that could be alleviated by the broadening of the range of agricultural compounds crops are resistant to. In addition, the broad resistance achieved here with plants expressing the yeast Tpolp transporter could allow farming in soils contaminated with heavy metals without altering the quality of the edible parts of crops, as the expression of this transporter did not lead to over-accumulation of toxic cations.

Homologs of the yeast Pdr5p in plants have proven pivotal in a wide range of processes such as xenobiotic detoxification, biotic stress resistance and phytohormone transport ${ }^{31}$. For example, AtPDR9 was shown to transport auxinic compounds such as the herbicide 2,4-D and the endogenous auxin precursor indole-3-butyric acid (IBA) ${ }^{32,33}$. AtPDR8 has also been shown to be involved in IBA transport as well as to play roles in the resistance to ions such as $\mathrm{Cd}^{2+}$ and $\mathrm{Na}^{+}$, and contribute to plant defence from pathogens ${ }^{34-38}$. AtPDR12, on the other hand, is involved in $\mathrm{Pb}^{2+}$ detoxification, resistance to the diterpenoid sclareol, and the uptake of the plant hormone abscisic acid (ABA) $)^{39-41}$. Although the involvement of MFS transporters in xenobiotic resistance has not been widely studied, and none of those closest to Tpolp in Arabidopsis has been characterized, there are reported examples of the importance of the MFS in plant resistance to a range of insults. For instance, AtZIF1 and AtZIF2 are both involved in the tolerance to the $\mathrm{Zn}^{2+}$ cation $^{42,43}$, AtZIFL1 confers resistance to 2,4-D and to auxin ${ }^{44}$, and AtZIFL2 is important in maintaining $\mathrm{Cs}^{2+}$ and $\mathrm{K}^{+}$homeostasis in Arabidopsis ${ }^{45}$. Finally, we show here that expression of the S. cerevisiae Tpolp and Pdr5p in Arabidopsis enlarges the plant toolkit to successfully endure the effects of adverse soil conditions.

The present work demonstrates that the introduction of a single gene can expand the plant resistance repertoire from 2,4-D to not only other herbicides such as barban and the closely-related alachlor and metolachlor, but also to other active ingredients like the fungicides mancozeb and actidione (cycloheximide) and to soil contaminants such as metal ions. To our knowledge, the effects of barban and mancozeb on Arabidopsis were explored here for the first time. The results reported in this study could serve as the basis for future biotechnological strategies to improve plant performance in an increasingly challenging environment.

\section{Methods}

Plant materials and growth conditions. The Arabidopsis thaliana (L.) Heynh., ecotype Columbia (Col0 ), was used as the wild type in all experiments. Plant transformation was achieved by the floral-dip method ${ }^{46}$ using Agrobacterium tumefaciens strain EHA105. Seeds were surface-sterilized and sown on Murashige and Skoog ${ }^{47}$ medium solidified with $0.8 \%$ agar, stratified for $3 \mathrm{~d}$, placed in a growth chamber and transferred to soil after 2-3 weeks. Plants were cultivated under long-day conditions (16-h light, $22^{\circ} \mathrm{C} / 8$-h dark, $\left.18^{\circ} \mathrm{C}\right)$ at $60 \% \mathrm{RH}$.

Generation of transgenic lines. To generate ScTPO1 (YLL028W) and ScPDR5 (YOR153W) expression constructs, each full coding sequence, from start to stop codon, was PCR-amplified (Supplementary Table S1) using yeast genomic DNA. The resulting 1761-bp and 4536-bp fragments, corresponding to the ScTPO1 and ScPDR5 genes, respectively, were independently inserted via the XhoI/SpeI restriction sites into the pBA002 plasmid. After agroinfiltration of the resulting constructs into wild-type plants, six and five independent transformants showing significant expression of ScTPO1 and ScPDR5, respectively, were recovered. 
Gene expression analyses. Standard RT-PCR analyses were conducted as previously described ${ }^{48}$ using $^{-}$ primers designed to detect ScTPO1, ScPDR5, and UBQ10 (UBIQUITIN10) expression (Supplementary Table S1). The results shown are representative of three independent experiments. Real-time RT-PCR was performed using specific primers (Supplementary Table S1) on the CFX384 Touch Real-Time PCR Detection System (Bio-Rad) using the Luminaris mix (Thermoscientific) according to the manufacturer's instructions. For each condition tested, two RNA extractions from different biological samples and two reverse transcription reactions for each biological repeat were performed. Data were processed using Q-Gene ${ }^{49}$ that took the respective primer efficiency into consideration.

Subcellular localisation studies. To generate Tpolp and Pdr5p protein fusions with YFP and GFP, each coding sequence, excluding the stop codon, was PCR-amplified (Supplementary Table S1) using yeast genomic DNA as a template and independently inserted under the control of the $35 \mathrm{~S}$ promoter via the XhoI/PacI restriction sites into the YFP- or GFP-tagged versions of the pBA002 plasmid. Ten stable transgenic lines displaying a strong fluorescence signal in the root were recovered upon transformation of wild-type plants with the Pro35S:ScTPO1-YFP construct, whereas no fluorescence signal was detected in the two isolated stable transgenic lines expressing significant expression of the ScPDR5-YFP fusion. Arabidopsis protoplasts were generated as previously described ${ }^{50}$ and transfected with the YFP constructs by polyethylene glycol (PEG) transformation $^{51}$. Transient coexpression of the GFP constructs with the tonoplast marker $\gamma$-Tonoplast Intrinsic Protein (TIP)-mCherry or the plasma-membrane marker Plasma membrane Intrinsic Protein 2A (PIP2A)-mCherry ${ }^{52}$ and the pBIN-NA construct ${ }^{53}$ in leaf abaxial epidermal cells of Nicotiana tabacum was performed via agroinfiltration using A. tumefaciens strain GV3101.

Yeast manipulations. The parental Saccharomyces cerevisiae strain BY4741 and the derived deletion mutants BY4741_ $\Delta$ tpo1 and BY4741_ $\Delta p d r 5$ previously described ${ }^{11}$ were used. Growth curve and spot assays were conducted in minimal growth medium MMB liquid or agarized ${ }^{12}$ and supplemented with the indicated compound (Sigma-Aldrich) at the desired concentration. Results presented are representative of three independent experiments.

Plant phenotypical assays. Root assays were performed as described by Remy and Duque ${ }^{14}$. Briefly, 5-d old seedlings grown vertically were transferred to fresh medium containing the indicated compound (Sigma-Aldrich) at the specified concentration. After additional 1 and 3 weeks of growth, PR elongation and shoot biomass/chlorophyll content, respectively, were evaluated. All assays were performed in a climate-controlled growth cabinet under the long-day conditions described above. Similar results were obtained in at least three independent experiments performed with different seed batches (one of which representing a line homozygous for the transgene insertion), and representative data from a single experiment are presented.

Microscopy. Confocal images were taken with an LSM 510 laser-scanning microscope equipped with a Meta detector (Zeiss, Germany). Excitation wavelengths used to detect fluorescence were $488 \mathrm{~nm}$ for GFP, $514 \mathrm{~nm}$ for YFP and $543 \mathrm{~nm}$ for mCherry. Emitted fluorescence was monitored at detection wavelengths between 565$615 \mathrm{~nm}$ for mCherry, between 535-590 nm for YFP and between 500-550 nm for GFP.

2,4-D accumulation assays. Radiolabeled [14C]2,4-D accumulation was assayed in root-tip segments as previously described ${ }^{5,32}$, except that the radiolabeled compound was used at a final concentration of $7.5 \mu \mathrm{M}$ (50 mCi/mmol) (American Radiolabeled Chemicals).

Metal ion content determination. To measure total ion content, pooled shoot tissues from 3-week old seedlings challenged with $0.5 \mathrm{mM} \mathrm{Al}^{3+}, 50 \mu \mathrm{M} \mathrm{Cd}^{2+}, 75 \mu \mathrm{M} \mathrm{Co}^{2+}, 50 \mu \mathrm{M} \mathrm{Cu}^{2+}$ and $100 \mu \mathrm{M} \mathrm{Ni}^{2+}$ were processed as previously described ${ }^{48}$. Concentration of the digests for $\mathrm{Al}^{3+}, \mathrm{Cd}^{2+}, \mathrm{Co}^{2+}, \mathrm{Cu}^{2+}$ and $\mathrm{Ni}^{2+}$ was quantified using the Atomic Emission Spectrometry - Inductively Coupled Plasma - Optical Emission System (Perkin-Elmer Optical Emission, Optima 2100 DV) at the Laboratório de Análises, Instituto Superior Técnico (Lisbon, Portugal) according to method SMEWW $3120 \mathrm{~B}$ described by Eaton et al. ${ }^{54}$. Standards for analytical calibration were from Merck KGaA (Germany), and four independent samples were processed per genotype.

Data Availability. All data generated or analysed during this study are included in this published article (and its Supplementary Information files).

\section{References}

1. Peuke, A. D. \& Rennenberg, H. Phytoremediation. EMBO reports 6, 497-501, doi:10.1038/sj.embor.7400445 (2005).

2. Zhu, B. et al. Enhanced transformation of TNT by Arabidopsis plants expressing an old yellow enzyme. PloS one 7, e39861, doi:10.1371/journal.pone.0039861 (2012).

3. Behrens, M. R. et al. Dicamba resistance: enlarging and preserving biotechnology-based weed management strategies. Science 316, 1185-1188, doi:10.1126/science.1141596 (2007).

4. Khoudi, H., Maatar, Y., Gouiaa, S. \& Masmoudi, K. Transgenic tobacco plants expressing ectopically wheat H+-pyrophosphatase (H+-PPase) gene TaVP1 show enhanced accumulation and tolerance to cadmium. Journal of plant physiology 169, 98-103, doi:10.1016/j.jplph.2011.07.016 (2012).

5. Khoudi, H. et al. Phytoremediation potential of Arabidopsis thaliana, expressing ectopically a vacuolar proton pump, for the industrial waste phosphogypsum. Environmental Science and Pollution Research 20, 270-280, doi:10.1007/s11356-012-1143-2 (2013).

6. Siemianowski, O. et al. HMA4 expression in tobacco reduces Cd accumulation due to the induction of the apoplastic barrier. Journal of experimental botany 65, 1125-1139, doi:10.1093/jxb/ert471 (2014).

7. Ali, W. et al. Heterologous expression of the yeast arsenite efflux system ACR3 improves Arabidopsis thaliana tolerance to arsenic stress. The New phytologist 194, 716-723, doi:10.1111/j.1469-8137.2012.04092.x (2012). 
8. Song, W.-Y. et al. In Biotechnology and Sustainable Agriculture 2006 and Beyond: Proceedings of the 11th IAPTCer B Congress, August 31-18, 2006 Beijing, China (eds Zhihong Xu, Jiayang Li, Yongbiao Xue, \& Weicai Yang) 265-271 (Springer Netherlands, 2007).

9. Sá-Correia, I., dos Santos, S. C., Teixeira, M. C., Cabrito, T. R. \& Mira, N. P. Drug:H+ antiporters in chemical stress response in yeast. Trends in microbiology 17, 22-31, doi:10.1016/j.tim.2008.09.007 (2009).

10. Prasad, R. \& Goffeau, A. Yeast ATP-Binding Cassette Transporters Conferring Multidrug Resistance. Annual Review of Microbiology 66, 39-63, doi:10.1146/annurev-micro-092611-150111 (2012).

11. Teixeira, M. C. \& Sa-Correia, I. Saccharomyces cerevisiae resistance to chlorinated phenoxyacetic acid herbicides involves Pdr1pmediated transcriptional activation of TPO1 and PDR5 genes. Biochemical and biophysical research communications 292, 530-537, doi:10.1006/bbrc.2002.6691 (2002).

12. Cabrito, T. R., Teixeira, M. C., Duarte, A. A., Duque, P. \& Sa-Correia, I. Heterologous expression of a Tpol homolog from Arabidopsis thaliana confers resistance to the herbicide 2,4-D and other chemical stresses in yeast. Applied microbiology and biotechnology 84, 927-936, doi:10.1007/s00253-009-2025-5 (2009).

13. Emter, R., Heese-Peck, A. \& Kralli, A. ERG6 and PDR5 regulate small lipophilic drug accumulation in yeast cells via distinct mechanisms. FEBS letters 521, 57-61, doi:10.1016/S0014-5793(02)02818-1 (2002).

14. Remy, E. \& Duque, P. In Environmental Responses in Plants: Methods and Protocols (ed Paula, Duque) 197-208 (Springer New York, 2016)

15. Desai, P. N., Shrivastava, N. \& Padh, H. Production of heterologous proteins in plants: Strategies for optimal expression. Biotechnology Advances 28, 427-435, doi:10.1016/j.biotechadv.2010.01.005 (2010).

16. Liu, L. \& Fan, X.-D. CRISPR-Cas system: a powerful tool for genome engineering. Plant molecular biology 85, 209-218, doi:10.1007/ s11103-014-0188-7 (2014).

17. Urnov, F. D., Rebar, E. J., Holmes, M. C., Zhang, H. S. \& Gregory, P. D. Genome editing with engineered zinc finger nucleases. Nature reviews. Genetics 11, 636-646 (2010).

18. Joung, J. K. \& Sander, J. D. TALENs: a widely applicable technology for targeted genome editing. Nat Rev Mol Cell Biol 14, 49-55, http://www.nature.com/nrm/journal/v14/n1/suppinfo/nrm3486_S1.html (2013).

19. Bissinger, P. H. \& Kuchler, K. Molecular cloning and expression of the Saccharomyces cerevisiae STS1 gene product. A yeast ABC transporter conferring mycotoxin resistance. Journal of Biological Chemistry 269, 4180-4186 (1994).

20. Kralli, A., Bohen, S. P. \& Yamamoto, K. R. LEM1, an ATP-binding-cassette transporter, selectively modulates the biological potency of steroid hormones. Proceedings of the National Academy of Sciences of the United States of America 92, 4701-4705 (1995).

21. Leppert, G. et al. Cloning by gene amplification of two loci conferring multiple drug resistance in Saccharomyces. Genetics 125, 13-20 (1990)

22. Kolaczkowski, M., Kolaczkowska, A., Luczynski, J., Witek, S. \& Goffeau, A. In Vivo Characterization of the Drug Resistance Profile of the Major ABC Transporters and Other Components of the Yeast Pleiotropic Drug Resistance Network. Microbial Drug Resistance 4, 143-158, doi:10.1089/mdr.1998.4.143 (1998).

23. Albertsen, M., Bellahn, I., Kramer, R. \& Waffenschmidt, S. Localization and function of the yeast multidrug transporter Tpolp. The Journal of biological chemistry 278, 12820-12825, doi:10.1074/jbc.M210715200 (2003).

24. Tomitori, H. et al. Multiple polyamine transport systems on the vacuolar membrane in yeast. The Biochemical journal 353, 681-688 (2001).

25. C W Tabor, A. \& Tabor, H. Polyamines. Annual Review of Biochemistry 53, 749-790, doi:10.1146/annurev.bi.53.070184.003533 (1984).

26. Pomorski, T. et al. Drs2p-related P-type ATPases Dnflp and Dnf2p are required for phospholipid translocation across the yeast plasma membrane and serve a role in endocytosis. Molecular biology of the cell 14, 1240-1254, doi:10.1091/mbc.E02-08-0501 (2003).

27. Sa-Correia, I., dos Santos, S. C., Teixeira, M. C., Cabrito, T. R. \& Mira, N. P. Drug:H+ antiporters in chemical stress response in yeast. Trends in microbiology 17, 22-31, doi:10.1016/j.tim.2008.09.007 (2009).

28. Ryan, P. R., Ditomaso, J. M. \& Kochian, L. V. Aluminium Toxicity in Roots: An Investigation of Spatial Sensitivity and the Role of the Root Cap. Journal of experimental botany 44, 437-446, doi:10.1093/jxb/44.2.437 (1993).

29. Grube, A., Donaldson, D., Kiely, T. \& Wu, L. Pesticides Industry Sales and Usage 2006 and 2007 Market Estimates (U.S. Environmental Protection Agency, Washington, DC, 2011).

30. Johnson, W. et al. 2,4-D- and Dicamba-tolerant Crops - Some Facts to Consider, https://www.extension.purdue.edu/extmedia/ID/ ID-453-W.pdf (2012).

31. Remy, E. \& Duque, P. Beyond cellular detoxification: a plethora of physiological roles for MDR transporter homologs in plants. Frontiers in physiology 5, 201, doi:10.3389/fphys.2014.00201 (2014).

32. Ito, H. \& Gray, W. M. A gain-of-function mutation in the Arabidopsis pleiotropic drug resistance transporter PDR9 confers resistance to auxinic herbicides. Plant physiology 142, 63-74, doi:10.1104/pp.106.084533 (2006).

33. Ruzicka, K. et al. Arabidopsis PIS1 encodes the ABCG37 transporter of auxinic compounds including the auxin precursor indole3-butyric acid. Proceedings of the National Academy of Sciences of the United States of America 107, 10749-10753, doi:10.1073/ pnas.1005878107 (2010).

34. Kim, D.-Y., Bovet, L., Maeshima, M., Martinoia, E. \& Lee, Y. The ABC transporter AtPDR8 is a cadmium extrusion pump conferring heavy metal resistance. The Plant Journal 50, 207-218, doi:10.1111/j.1365-313X.2007.03044.x (2007).

35. Strader, L. C. \& Bartel, B. The Arabidopsis PLEIOTROPIC DRUG RESISTANCE8/ABCG36 ATP Binding Cassette Transporter Modulates Sensitivity to the Auxin Precursor Indole-3-Butyric Acid. The Plant cell 21, 1992-2007, doi:10.1105/tpc.109.065821 (2009).

36. Underwood, W. \& Somerville, S. C. Perception of conserved pathogen elicitors at the plasma membrane leads to relocalization of the Arabidopsis PEN3 transporter. Proceedings of the National Academy of Sciences 110, 12492-12497, doi:10.1073/pnas.1218701110 (2013).

37. Stein, M. et al. Arabidopsis PEN3/PDR8, an ATP Binding Cassette Transporter, Contributes to Nonhost Resistance to Inappropriate Pathogens That Enter by Direct Penetration. The Plant cell 18, 731-746, doi:10.1105/tpc.105.038372 (2006).

38. Kim, D.-Y., Jin, J.-Y., Alejandro, S., Martinoia, E. \& Lee, Y. Overexpression of AtABCG36 improves drought and salt stress resistance in Arabidopsis. Physiologia plantarum 139, 170-180, doi:10.1111/j.1399-3054.2010.01353.x (2010).

39. Kang, J. et al. PDR-type ABC transporter mediates cellular uptake of the phytohormone abscisic acid. Proceedings of the National Academy of Sciences 107, 2355-2360, doi:10.1073/pnas.0909222107 (2010).

40. Campbell, E. J. et al. Pathogen-Responsive Expression of a Putative ATP-Binding Cassette Transporter Gene Conferring Resistance to the Diterpenoid Sclareol Is Regulated by Multiple Defense Signaling Pathways in Arabidopsis. Plant physiology 133, 1272-1284, doi:10.1104/pp.103.024182 (2003)

41. Lee, M., Lee, K., Lee, J., Noh, E. W. \& Lee, Y. AtPDR12 Contributes to Lead Resistance in Arabidopsis. Plant physiology 138, 827-836, doi:10.1104/pp.104.058107 (2005)

42. Haydon, M. J. \& Cobbett, C. S. A Novel Major Facilitator Superfamily Protein at the Tonoplast Influences Zinc Tolerance and Accumulation in Arabidopsis. Plant physiology 143, 1705-1719, doi:10.1104/pp.106.092015 (2007).

43. Remy, E. et al. Intron Retention in the 5'UTR of the Novel ZIF2 Transporter Enhances Translation to Promote Zinc Tolerance in Arabidopsis. PLoS genetics 10, e1004375, doi:10.1371/journal.pgen.1004375 (2014).

44. Remy, E. et al. A major facilitator superfamily transporter plays a dual role in polar auxin transport and drought stress tolerance in Arabidopsis. The Plant cell 25, 901-926, doi:10.1105/tpc.113.110353 (2013). 
45. Remy, E. et al. The major facilitator superfamily transporter ZIFL2 modulates cesium and potassium homeostasis in Arabidopsis. Plant \& cell physiology 56, 148-162, doi:10.1093/pcp/pcu157 (2015).

46. Clough, S. J. \& Bent, A. F. Floral dip: a simplified method for Agrobacterium-mediated transformation of Arabidopsis thaliana. The Plant journal: for cell and molecular biology 16, 735-743 (1998).

47. Murashige, T. \& Skoog, F. A Revised Medium for Rapid Growth and Bio Assays with Tobacco Tissue Cultures. Physiologia plantarum 15, 473-497, doi:10.1111/j.1399-3054.1962.tb08052.x (1962).

48. Remy, E. et al. The Pht1;9 and Pht1;8 transporters mediate inorganic phosphate acquisition by the Arabidopsis thaliana root during phosphorus starvation. The New phytologist 195, 356-371, doi:10.1111/j.1469-8137.2012.04167.x (2012).

49. Simon, P. Q-Gene: processing quantitative real-time RT-PCR data. Bioinformatics 19, 1439-1440 (2003).

50. Yoo, S. D., Cho, Y. H. \& Sheen, J. Arabidopsis mesophyll protoplasts: a versatile cell system for transient gene expression analysis. Nature protocols 2, 1565-1572, doi:10.1038/nprot.2007.199 (2007).

51. Abel, S. \& Theologis, A. Transient transformation of Arabidopsis leaf protoplasts: a versatile experimental system to study gene expression. Plant J 5, 421-427 (1994).

52. Nelson, B. K., Cai, X. \& Nebenfuhr, A. A multicolored set of in vivo organelle markers for co-localization studies in Arabidopsis and other plants. The Plant journal: for cell and molecular biology 51, 1126-1136, doi:10.1111/j.1365-313X.2007.03212.x (2007).

53. Silhavy, D. et al. A viral protein suppresses RNA silencing and binds silencing-generated, 21- to 25-nucleotide double-stranded RNAs. EMBO J 21, 3070-3080, doi:10.1093/emboj/cdf312 (2002).

54. Eaton, A. D., Clesceri, L. S., Rice, E. W. \& Greenberg, A. E. (American Public Health Association, American Water Works Association, Water Environment Federation, 2005).

\section{Acknowledgements}

We thank N.-H. Chua for the pBA002 vectors, M. Baião and V. Nunes for technical assistance, as well as R. Carvalho for photographing the plant phenotypes. This work was funded by Fundação para a Ciência e a Tecnologia (FCT) through grants EXPL/AGR-PRO/1013/2013 and PTDC/BIA-PLA/1084/2014 as well as fellowships SFRH/BPD/44640/2008, SFRH/BPD/81221/2011, PD/BD/105735/2014 (within the scope of the PhD program Molecular Biosciences PD/00133/2012) and SFRH/BD/92552/2013 awarded to E.R., T.R.C., M.N.-G, and C.P.G., respectively. The iBB - Institute for Bioengineering and Biosciences acknowledges funding from the Programa Operacional Regional de Lisboa 2020 (Project N. 007317) and the FCT (UID/BIO/04565/2013). Funding from the research unit GREEN-it "Bioresources for Sustainability" (UID/Multi/04551/2013) is also acknowledged.

\section{Author Contributions}

E.R., M.C.T., I.S.-C. and P.D. designed the research, and E.R., C.P.G. and T.R.C. performed the experiments. E.R., M.N.-G., M.C.T., I.S.-C. and P.D. analysed the data, and the article was written by M.N.-G. and P.D. with contributions from E.R. All authors discussed the results and contributed to the final manuscript.

\section{Additional Information}

Supplementary information accompanies this paper at doi:10.1038/s41598-017-04534-7

Competing Interests: The authors declare that they have no competing interests.

Publisher's note: Springer Nature remains neutral with regard to jurisdictional claims in published maps and institutional affiliations.

(c) (i) Open Access This article is licensed under a Creative Commons Attribution 4.0 International

License, which permits use, sharing, adaptation, distribution and reproduction in any medium or format, as long as you give appropriate credit to the original author(s) and the source, provide a link to the Creative Commons license, and indicate if changes were made. The images or other third party material in this article are included in the article's Creative Commons license, unless indicated otherwise in a credit line to the material. If material is not included in the article's Creative Commons license and your intended use is not permitted by statutory regulation or exceeds the permitted use, you will need to obtain permission directly from the copyright holder. To view a copy of this license, visit http://creativecommons.org/licenses/by/4.0/.

(c) The Author(s) 2017 